\title{
Agencia de Autocuidado y Adherencia al Tratamiento en Personas con Factores de Riesgo Cardiovascular
}

\section{Self-care agency and adherence to treatment in people having cardiovascular risk factors}

Anita Velandia-Arias y Luz N. Rivera-Álvarez

1 Unidad de Cuidado Intermedio, Hospital Tunjuelito. Bogotá, Colombia. E-mail: anita_velandia@hotmail.com 2 Facultad de Enfermería, Universidad Nacional de Colombia. Bogotá. E-mail: Inriveraa@unal.edu.co

Recibido 5 Diciembre 2008/Enviado para Modificación 3 Julio 2009/Aceptado 10 Julio 2009

\section{RESUMEN}

Objetivo Se determino la relación existente entre la Agencia de autocuidado con la adherencia al tratamiento farmacológico y no farmacológico en pacientes con algún factor de riesgo cardiovascular del programa de crónicos del hospital Tunjuelito.

Métodos Diseño descriptivo correlacional que midió y relacionó la Agencia de autocuidado y la Adherencia a los tratamientos en una muestra de 201 personas que contaban con algún factor de riesgo cardiovascular: Obesidad, Sedentarismo, Hipertensión arterial y Diabetes Mellitus, del Programa de crónicos del Hospital Tunjuelito. Se empleo la escala Apreciación de la agencia de autocuidado (ASA) y el Cuestionario para evaluar el comportamiento de adherencia a tratamiento farmacológico y no farmacológico derivado de la taxonomía NOC (Nursing Outcomes Clasification). Resultados El 62,6 \% de las personas con factores de riesgo cardiovascular tuvo regular Capacidad de agencia de autocuidado y el $77 \%$ de los participantes presentó una buena adherencia al tratamiento farmacológico y no farmacológico. La correlación resultante entre la Agencia de autocuidado y la Adherencia al tratamiento farmacológico y no farmacológico mostró una correlación moderada y positiva de 0.413 .

Conclusiones La agencia de autocuidado es un proceso de participación dinámico y tácito del individuo que le permite discernir, tomar decisiones y realizar acciones de autocuidado para el mantenimiento y mejora de su salud, entre ellas, la adherencia a los tratamientos.

Palabras Clave: Educación en Enfermería, enfermería en salud pública, atención de enfermería, investigación en enfermería, autocuidado; factores de riesgo, participación del paciente (fuente: DeCS, BIREME).

\section{ABSTRACT}

Objective Determining the relationship between self-care agency and adherence to pharmacological and non-pharmacological treatment in patients having cardiovascular risk factors at the Tunjuelito hospital in Bogota. 
Methodology This study involved a descriptive and correlational design which evaluated self-care agency ability and adherence to treatment in a random sample of 201 people having the following cardiovascular risk factors: being obese, having a sedentary lifestyle, arterial hypertension and diabetes mellitus. The patients being studied were attending Tunjuelito hospital's chronic-patient programme. Appraisal of self-care agency (ASA) scale and a questionnaire for evaluating behaviour regarding adherence to pharmacological and non-pharmacological treatment from the NOC taxonomy were carried out in the research.

Results $62.6 \%$ of the people having cardiovascular risk factors had regular self-care agency ability, whilst $77 \%$ presented good adherence to pharmacological and nonpharmacological treatment. Spearman's coefficient revealed a 0.413 correlation between self-care agency ability and adherence to pharmacological and non-pharmacological treatment, indicating moderate and positive correlation.

Conclusions Self-care agency involves an individual's active and tacit participation in discerning, decision-making and taking self-care action for maintaining and improving her/his health (i.e. adhering to prescribed treatment).

Key Words: Education, nursing, public health nursing, nursing care, nursing research, model, nursing, self-care, risk-factor, patient participation, patient compliance, treatment outcome (source: MeSH, NLM).

$\mathrm{E}$ 1 aumento de enfermedades crónicas, tales como enfermedades cardiovasculares (enfermedad isquémica del corazón, enfermedad cerebro vascular, hipertensión), cáncer, enfermedades pulmonares crónicas, entre otras, está determinado por los estilos de vida (1), la capacidad que tiene el individuo para comprometerse con su propio cuidado, y la adherencia a los tratamientos, tanto farmacológicos como no farmacológicos.

Las enfermedades cardiovasculares son síntomas (marcas) propios de la actual sociedad ("moderna- postmoderna"), cuyos índices de morbi mortalidad van cada día en incremento, y son reconocidas como entidades crónicas y de lento desarrollo, éstas son producidas cuando confluye un número suficiente de factores desencadenantes o "factores de riesgo". Por lo tanto, cobra importancia la capacidad que tiene el individuo de ser agente de su propio cuidado, como forma de promoción y prevención tanto de los factores de riesgo como de las enfermedades cardiovasculares.

Esta capacidad del individuo es lo que denomina Orem (2) como Capacidad de agencia de autocuidado, que implica un proceso de participación dinámico por parte de las personas en el cuidado de su propia salud. Proceso mediante el cual la persona discierne sobre los factores que deben ser controlados o tratados para autorregularse, decide lo que puede y debería hacer con respecto a ésta regulación, valora y reflexiona sobre sus capacidades específicas para 
comprometerse -implícita aquí la responsabilidad- en el cuidado de su propia salud, y finalmente realiza acciones psicomotoras. El objetivo principal de estas decisiones y acciones reflexionadas es satisfacer las necesidades de autocuidado a lo largo del tiempo y/o en situaciones de alteración del estado de salud.

Estas necesidades pueden ser de tipo universal como: aporte de alimento, agua y aire adecuado, equilibrio entre la actividad y el reposo, equilibrio entre soledad e interacción social, prevención de riesgos, promoción del funcionamiento y desarrollo humano. Necesidades relativas al desarrollo y maduración, en las que involucra además la promoción de condiciones y experiencias para facilitar el desarrollo de prácticas intelectuales, interaccionales y sociales, esenciales para vivir en sociedad. Y por último, necesidades relacionadas con el estado de salud de la persona y su tratamiento, como monitorización de signos y síntomas, adherencia al tratamiento farmacológico y no farmacológico, entre otros.

La capacidad del individuo para satisfacer estas necesidades requiere de tres tipos de capacidades. Las primeras son capacidades fundamentales y de disposición como la sensación, percepción, memoria y orientación. Las segundas son capacidades específicas que se relacionan con la habilidad del individuo para comprometerse en el autocuidado, la valoración de la salud, energía y conocimiento del autocuidado; éstas son: máxima atención y vigilancia, uso controlado de la energía y del cuerpo, adquisición de conocimiento, razonamiento, motivación y toma de decisiones. Por último, capacidades que le permitan operacionalizar las decisiones, donde el individuo investiga condiciones y factores de sí mismo y del medio ambiente que son significativas para su autocuidado, toma juicios y decisiones -auto reflexionadas- de autocuidado y construye medidas para satisfacer los requisitos de autocuidado por sí mismo (3).

Una de esas medidas ya operacionalizadas es la adherencia a los tratamientos. De acuerdo con la OMS, la adherencia "es el grado en que el comportamiento de una persona -tomar el medicamento, seguir un régimen alimentario y ejecutar cambios del modo de vida- se corresponde con las recomendaciones acordadas de un prestador de asistencia sanitaria" (4).

Este comportamiento humano implica compromiso y responsabilidad de las personas con el cuidado y mantenimiento de su salud. La adherencia es un factor importante susceptible de ser modificable y que compromete los resultados en los tratamientos instaurados: la falta de adherencia a los tratamientos farmacológicos y a las recomendaciones sobre cambios en el estilo de vida, conduce a un comportamiento contrario al autocuidado. 
La falta de adherencia se da por una interrelación de factores entre los cuales se encuentran algunos derivados de la persona y su entorno económico, cultural y social; otros derivados de la enfermedad en sí, del tratamiento farmacológico y sus características, así como los derivados de los servicios de salud y del personal que atiende al paciente.

La adherencia comprende acciones de autocuidado complejas que resultan de recibir orientación acerca de los tratamientos farmacológicos y no farmacológicos que implica cambios en el estilo de vida. Para su éxito requiere de procesos cognitivos de razonamiento y toma de decisiones reflexionadas. Por lo tanto el estudio busco determinar la relación existente entre la Capacidad de agencia de autocuidado con la adherencia al tratamiento farmacológico y no farmacológico en pacientes que presentaron algún factor de riesgo cardiovascular y que participaron en el Programa de Crónicos del Hospital Tunjuelito.

\section{METODOS Y MATERIALES}

Estudio descriptivo correlacional que evaluó y relacionó la agencia de autocuidado y la adherencia a los tratamientos (farmacológicos y no farmacológicos) en una muestra de 201 personas que contaban con algún factor de riesgo cardiovascular como: hipertensión arterial, diabetes, obesidad y sedentarismo del Programa de Crónicos del Hospital Tunjuelito. Este programa contaba con una población total de 812 personas, los participantes fueron seleccionados por muestreo probabilístico estratificado y la recolección de datos fue realizada en los meses de mayo a julio de 2007.

Los criterios de inclusión del estudio fueron: mujeres y hombres mayores de 18 años con algún factor de riesgo cardiovascular, persona consiente que pueda comunicarse y participación voluntaria en el estudio.

\section{Instrumentos}

Escala "Apreciación de la agencia de autocuidado"ASA. La escala ASA ha sido desarrollada para medir las capacidades de agencia de autocuidado El desarrollo de la escala fue un trabajo colectivo de profesores de la Wayne State University y la University of Limburg (5). La escala esta basada en la Teoría del Déficit de Autocuidado de Dorothea Orem. Y ha sido traducida, adaptada transculturalmente y validada para países de Europa, Asia oriental y Latinoamérica (6). 
La medición de la validez conceptual y confiabilidad de la escala ASA en versión español fue realizada por Gallegos en un grupo de pacientes con diabetes, obteniendo un alfa de Cronbach de 0,77 (7). Ésta escala fue revisada y adaptada transculturalmente para la población Colombiana por la profesora Edilma de Reales de la Facultad de Enfermería de la Universidad Nacional de Colombia, con un coeficiente de confiabilidad alfa de Cronbach de 0.7440 (8). La escala ASA consta de 24 ítems tipo Likert de cuatro alternativas de respuesta: uno (nunca) significa el valor más bajo de la agencia de autocuidado y cuatro (siempre) el más alto.

Cuestionario de valoración del Comportamiento de Adherencia al tratamiento farmacológico y no farmacológico. Éste cuestionario en su versión original, pasó por el proceso de validez de contenido por enfermeras expertas tomando como referente los indicadores y la escala semántica de la Taxonomía NOC (Nursing Outcomes Classification, Clasificación de los resultados de enfermería) de la universidad de IOWA 1997, empleando el modelo de evaluación propuesto por Richard Fehring (9).

El cuestionario en versión español fue aplicado por Bastidas en personas con alguna condición de enfermedad coronaria (10). Consta de 14 ítems tipo Likert de cinco alternativas de respuesta donde uno (nunca) significa el valor más bajo de la adherencia a los tratamientos y cinco (siempre) el más alto. Además cuenta con una guía anexa de preguntas, que permite confrontar la información suministrada por el paciente con datos de la historia clínica, como son el peso, la tensión arterial, entre otros, y con la información del cuidador del paciente.

Procedimiento. Previo al inicio de recolección de datos se explicó a cada participanteel objetivo del estudio, los principios éticos de autonomía, confidencialidad, veracidad de la información y el derecho a retirarse cuando la persona lo determinara, y por último se obtuvo su consentimiento informado por escrito para participar.

Para la recolección de la información se contó con una sala dispuesta para reuniones educativas en cada sede del programa Crónicos del Hospital donde se midió la tensión arterial, el peso, la talla y el nivel de actividad física a cada participante. Posteriormente, en la mayoría de casos cada participante auto diligenció la Escala "Apreciación de la agencia de autocuidado"-ASA. El "Cuestionario de valoración del Comportamiento de Adherencia al tratamiento farmacológico y no farmacológico" fue aplicado directamente por el grupo investigador al paciente, confrontando la información con la guía anexa de éste cuestionario y con datos del cuidador, cuando estaba presente. 
Para establecer la asociación estadística entre agencia de autocuidado y adherencia a los tratamientos farmacológicos y no farmacológicos se empleó el coeficiente de Spearman. La información fue analizada empleando el programa SPSS v.12 y para el análisis entre variables se empleó el programa SPAD.

\section{RESULTADOS}

El $78 \%(n=157)$ de los participantes eran mujeres y el $22 \%(n=44)$ eran hombres, la edad promedio de los participantes fue de 65.6, cuyo rango de edad estuvo entre 36 años y 89 años (DS 11,3). Los resultados mostraron que existió predominio de los factores de riesgo cardiovascular en las personas mayores de 50 años, el $9 \%$ de los participantes pertenecían al grupo de 35 a 49 años, el $36 \%$ al rango de edad de 50 a 64 años, el $43 \%$ al rango de 65 a 79 años y el $12 \%$ al rango de 80 años y más.

Respecto a las condiciones socioeconómicas, el $62 \%$ de la población era del estrato 2 y el $35 \%$ del estrato 1 . La ocupación que destacó en los participantes fue la estancia en el hogar con un $85,6 \%$, oficios varios fue desempeñada en un $3,5 \%$, conductores en un $3 \%$, comerciantes y modistas con el 2,5\%, ebanistería con el $2 \%$ y constructores e independientes con el $0,5 \%$.

En cuanto a la escolaridad ninguno de los participantes superó el grado de primaria básica. La educación al igual que las condiciones socioeconómicas tiene un impacto en la salud a través de su influencia sobre las conductas de estilo de vida, capacidad para realizar procesos autoreflexivos, tomar decisiones, resolver problemas, entre otros.

Perfil clínico de los participantes

El 58,2 \% (n=117) de los participantes presentaron tres factores de riesgo cardiovascular, el 33,3\% (n=67) presentaron dos de los factores de riesgo, el $8 \%$ $(\mathrm{n}=16)$ presentaron los cuatro factores de riesgo y sólo el $0,5 \%(\mathrm{n}=1)$ de las personas presentó un factor de riesgo cardiovascular.

Los datos arrojados por factor de riesgo evidencia la prevalencia de hipertensión arterial en los participantes. El 91,5\% $(\mathrm{n}=184)$ presento hipertensión arterial, el $6,9 \%(\mathrm{n}=14)$ hipertensión arterial más diabetes mellitus, el $1 \%(\mathrm{n}=2)$ diabetes mellitus, el $96 \%(n=193)$ sedentarismo, el $23 \%(n=47)$ obesidad, el $43 \%(n=85)$ sobrepeso, además en el estudio se encontró que el 1,4 \% $(n=3)$ se encontraba en desnutrición. 
De acuerdo al número de medicamentos prescrito, el $62 \%$ de las personas tomaba de 1 a 2 medicamentos, el $37 \%$ de 3 a 4 medicamentos y sólo el $1 \%$ tomaba cinco o más medicamentos.

Los factores de riesgo están asociados a estilos de vida, sin embargo la oportunidad para que el individuo ejecute hábitos saludables no son simplemente selecciones libres acerca de estilos de vida, son decisiones tomadas desde las alternativas de las que dispone el individuo de acuerdo a sus circunstancias socioeconómicas y la capacidad de elegir una opción y no otra.

Capacidad de agencia de autocuidado y Adherencia al tratamiento

La media resultante de la capacidad de agencia de autocuidado en los participantes correspondió a 78,5 (DS 6,6), con un rango de respuesta entre 57 a 92 puntos, frente a un rango real de la escala de 24 a 96 puntos.

Según los resultados arrojados en la escala ASA se encontró que el $35 \%$ de los participantes presento buena agencia de autocuidado, el $63 \%$ presento regular agencia de autocuidado y el $1 \%$ tuvo baja agencia de autocuidado. En las Tablas 1 y 2 se presentan los ítems de la escala ASA que obtuvieron mayor y menor puntuación respectivamente.

La media resultante de la adherencia al tratamiento fue de 59,6 (DS 4,9), los valores de respuesta de los participantes oscilaron entre 45 y 70 puntos, frente al rango real del cuestionario de 14 a 70 puntos. El $77 \%$ de las personas presento buena adherencia al tratamiento farmacológico y no farmacológico y el $23 \%$ tuvo regular adherencia. Se observo una tendencia en las dos escalas hacia las respuestas 'casi siempre' y 'siempre'.

Los comportamientos de adherencia que presentaron los participantes fueron:

- El $94 \%$ de las personas solicita el tratamiento que se le ha prescrito

- El $93 \%$ de los participantes cumple las citas del profesional de salud

- El 92,5\% de las personas informa que esta cumpliendo el tratamiento prescrito (hace referencia a tratamiento farmacológico)

- El 91,5\% de los participantes aceptan el diagnóstico que le da el profesional de salud. 
Los comportamientos de adherencia que presentan mayor dificultad en las personas del estudio están relacionados con el tratamiento no farmacológico:

- El $48 \%$ de las personas reportan que nunca o casi nunca han dejado de fumar o disminuir la cantidad de cigarrillos al día.

- El 23,3 \% de las personas siempre logra mantener el peso dentro de límites recomendados.

- El 27,8 \% de los participantes siempre mantiene su tensión arterial dentro de los límites deseables.

- El 31,8 \% informa que siempre hace el ejercicio recomendado

Tabla 1. Ítems de la escala ASA con mayor puntuación en personas con factores de riesgo cardiovascular

\begin{tabular}{|c|c|c|}
\hline Ítem & $\begin{array}{l}\text { Frecuencia } \\
(\mathrm{n})\end{array}$ & $\begin{array}{l}\text { Porcentaje } \\
(\%)\end{array}$ \\
\hline $\begin{array}{l}\text { Hago en primer lugar lo que sea necesario para } \\
\text { mantenerme con salud. }\end{array}$ & 159 & 79,1 \\
\hline $\begin{array}{l}\text { Cambio la frecuencia con que me baño para } \\
\text { mantenerme limpio. }\end{array}$ & 145 & 72,1 \\
\hline $\begin{array}{l}\text { Cuando obtengo información sobre mi salud pido } \\
\text { explicaciones sobre lo que no entiendo }\end{array}$ & 147 & 73,1 \\
\hline $\begin{array}{l}\text { Cuando tengo que tomar una nueva medicina cuento } \\
\text { con una persona que me brinda información sobre } \\
\text { los efectos secundarios }\end{array}$ & 134 & 66,6 \\
\hline $\begin{array}{l}\text { Si mi salud se ve afectada yo puedo conseguir la } \\
\text { información necesaria sobre que hacer. }\end{array}$ & 140 & 69,6 \\
\hline Si yo no puedo cuidarme puedo buscar ayuda. & 125 & 62,1 \\
\hline
\end{tabular}

Tabla 2. Ítems de la escala ASA con menor puntuación en personas con factores de riesgo cardiovascular

\begin{tabular}{lcc}
\multicolumn{4}{c}{ IItem } & \multicolumn{1}{c}{\begin{tabular}{c}
\multicolumn{1}{c}{ factores de riesgo cardiovascular } \\
\hline
\end{tabular}} & $\begin{array}{c}\text { Frecuencia } \\
(\mathrm{n})\end{array}$ & $\begin{array}{c}\text { Porcentaje } \\
(\%)\end{array}$ \\
\hline $\begin{array}{l}\text { Me faltan las fuerzas necesarias para cuidarme } \\
\text { como debo }\end{array}$ & 48 & 23,3 \\
$\begin{array}{l}\text { Pienso en hacer ejercicio y descansar un poco } \\
\text { durante el día pero no llego hacerlo. }\end{array}$ & 28 & 16,4 \\
$\begin{array}{l}\text { Debido a mis ocupaciones diarias me resulta } \\
\text { difícil sacar tiempo para cuidarme }\end{array}$ & 44 & 19,4 \\
$\begin{array}{l}\text { Cuando necesito ayuda puedo recurrir a mis } \\
\text { amigos de siempre }\end{array}$ & 63 & 31,3 \\
$\begin{array}{l}\text { Para mantener el peso que me corresponde } \\
\text { hago cambios en mis hábitos alimentarios. }\end{array}$ & 65 & 32,3 \\
\hline
\end{tabular}


En el análisis factorial multivariante (utilizando el programa SPAD) se encontró una asociación fuerte de la Agencia de autocuidado y la Adherencia a los tratamientos en las siguientes variables: mujeres, personas con ocupación hogar y personas en el rango de edad de 50 a 64 años. Los hombres y el grupo etáreo de 80 y más años presentaron tendencia a regular Agencia de autocuidado y regular adherencia a los tratamientos. Con respecto al número de medicamentos se encontró relación entre ingesta de 2 y 3 medicamentos con regular Agencia de autocuidado y regular adherencia a tratamientos. Y por último, el grupo de edad de 35 a 49 años y las personas que ocupan otras ocupaciones no presentaron asociación con la agencia de autocuidado y con la adherencia a los tratamientos.

Lo anterior permite concluir que la realización de su ocupación, puede ser un factor contributivo para que la persona deje de lado el compromiso para auto cuidarse.

Por último, al analizar los valores obtenidos de la muestra respecto a la agencia de autocuidado y la adherencia al tratamiento farmacológico y no farmacológico resultó un valor del coeficiente de Spearman de 0.413 esto indica una correlación moderada con tendencia positiva.

\section{DISCUSIÓN}

Los resultados del estudio corroboran la teoría del déficit de autocuidado de Orem (14), quien identificó que el estado de salud es un factor básico condicionante que influye en la agencia de autocuidado. Lo anterior se evidencia en que más de la mitad de los participantes presento regular agencia de autocuidado, contaban con factores de riesgo cardiovascular y presentaron insatisfacción en necesidades de tipo universal como: 'el mantenimiento del equilibrio entre la actividad y el reposo' y 'la ingesta de alimentos adecuada para lograr mantener la persona su peso ideal'. Resultados similares a los de Rivera (11), en pacientes hospitalizados con hipertensión arterial.

Respecto a la escala ASA, los ítems que presentan más baja puntuación en los participantes están los relacionados con capacidades específicas de la agencia de autocuidado que menciona Dorothea Orem, como son: Uso controlado de la energía física, para la iniciación y continuación de las operaciones de autocuidado, toma de decisiones sobre autocuidado y la operacionalización de estas decisiones. Esto se corrobora con los resultados del Cuestionario de adherencia al tratamiento farmacológico y no farmacológico en los participantes del estudio, quienes en gran porcentaje presentaron mayor dificultad para adherirse a tratamientos de 
tipo no farmacológico como: dejar de fumar, mantener el peso dentro de límites recomendados, mantener su tensión arterial dentro de límites deseables y hacer el ejercicio recomendado.

Martínez de D. et al., (12) en un estudio cuasi experimental, aplicaron un modelo educativo de autocuidado a pacientes hospitalizados con diabetes mellitus II; los pacientes del grupo experimental tuvieron una media menor de días estancia en la institución y menores reingresos respecto al grupo control, incrementó el conocimiento relacionado con la enfermedad y el tratamiento, así como las acciones de autocuidado, lo cual se reflejó en un descenso significativo en los niveles de hemoglobina glucosilada. La aplicación del modelo educativo logró disminuir la proporción de pacientes con tratamiento de insulina, previa indicación médica y se cambió al tratamiento con hipoglucemiantes orales, junto con la adherencia a la dieta y al ejercicio. De los pacientes que continuaron con el tratamiento de insulina, todas se la autoadministraban al finalizar la intervención, y el resto que continuó con dicho tratamiento recibió apoyo de algún miembro de la familia.

Lo anterior ratifica la importancia de la educación en salud orientada al individuo y a la familia. La familia cumple un papel mediador y de soporte entre la agencia de autocuidado y las acciones de autocuidado del individuo. De igual manera, la OMS (13) sugiere que las acciones de autocuidado especialmente en Estados de desviación de la salud están relacionadas con el conocimiento que se tiene de la patología y del manejo adecuado del mismo para tener una adaptación acorde con su nueva situación de salud y adherirse eficazmente a los tratamientos.

Con respecto a la adherencia se mostró alta en los ítems afines con la relación con el equipo de salud y su credibilidad en los mismos para aceptar y cumplir los tratamientos ordenados. Cáceres (15) considera primordial las características de la enfermedad en sí misma y la relación asertiva entre médico y paciente para proveer adherencia, la adecuada comunicación entre el personal de salud y el paciente esta descrita como un factor que determina la adherencia.

Los resultados muestran una correlación moderada con tendencia positiva de 0.413 , lo que significa que cuando el individuo posee mejores capacidades para autocuidarse, cuenta con mayores habilidades para adherirse a los tratamientos, ya sea farmacológico o no farmacológico. Resultado similar al reportado por Bastidas, quien en su estudio con personas con alguna condición de enfermedad coronaria, mostró una correlación directa y moderada de 0.515 entre la agencia de autocuidado y la adherencia. 
La agencia de autocuidado es un proceso de participación dinámico y tácito del individuo que le permite discernir, tomar decisiones y realizar acciones de autocuidado para el mantenimiento y mejora de su salud, entre ellas, la adherencia a los tratamientos .

\section{REFERENCIAS}

1. Mejía EN, Serna LE, Sierra T, Muñoz A. Descripción de actitudes y prácticas de estilo de vida saludables en médicos y personal de enfermería, Metrosalud, Medellín, Antioquia, 1997. Rev Epidemiol Antioquia 1999; 24(3-4):195-206.

2. Orem DE. Modelo de Orem. Conceptos de enfermería en la práctica. Barcelona: Masson Salvat; 1993.

3. Gast H, Denyes M, Campbell J, Hatweg D, Schott-Baer D, Isenberg MA. Self care agency: conceptualizations and operationalizations. Adv Nurs Sci 1989; 12(1):26-38.

4. Sabaté E. Adherencia a los tratamientos a largo plazo: Pruebas para la acción. Organización Mundial de la Salud OMS; 2004. [Internet]. Disponible en: http://www.paho.org/Spanish/ AD/DPC/NC/adherencia-largo-plazo.pdf Consultado noviembre del 2008.

5. Evers GC, Isenberg MA, Philipsen H, Senten M, Brouns G. Validity testing of the Dutch translation of the appraisal of the self-care agency A.S.A.-scale. Int J Nurs Stud 1993; 30(4):331342.

6. Isenberg MA. Self-Care deficit theory of nursing: directions for advancing nursing and professional practice science. In: Parker M. Nursing Theories and Nursing practice. Philadelphia: F.A. Davis co; 2001. p.125-29.

7. Gallegos CE. Validez y confiabilidades de la versión en español de la escala: Valoración de las Capacidades de Autocuidado. Desarrollo Científ Enferm 1998; 6(9):260-266.

8. Velandia A, Rivera LN. Confiabilidad de la escala Apreciación de la agencia de autocuidado (ASA), segunda versión en español, adaptada para población colombiana. Av enferm 2009; 27(1):38-47.

9. Moorhead S, Johnson M, Maas M. Nursing outcomes Classification (NOC). San Louis: Mosby; 1997.

10. Bastidas CV. Asociación entre la capacidad de la agencia de autocuidado y la adherencia a tratamientos farmacológicos y no farmacológicos en personal con alguna condición de enfermedad coronaria. Av enferm 2007; 25(2):65-75.

11. Rivera LN. Capacidad de agencia de autocuidado en Personas con Hipertensión arterial hospitalizadas en una Clínica de Bogotá, Colombia. Rev. salud pública 2006; 8(3):235247.

12. Martínez MG, Moreno MG, Sauceda PF, Vázquez L, Medina SG, Liñán A. Modelo de manejo de casos en pacientes con diabetes mellitus tipo 2. Invest Educ Enferm 2006; 24(1):58-65.

13. OMS. Centro Para el desarrollo de la fármaco epidemiología. Sección de investigaciones. Caracterización de la información que le brinda el médico al paciente sobre el medicamento prescrito. Marianao 2005 [Internet]. Disponible en: http://www.cdf.sld.cu/Investigaciones/ prescripcion.marianao.pdf Consultado noviembre del 2007.

14. Orem DE. Soins infirmiers: les concepts et la pratique. $3^{\mathrm{a}}$ ed. Montreal: Décarie Éditeur inc; 1987. p.96.

15. Cáceres MF. Factores de riesgo para abandono (no adherencia) del tratamiento antituberculoso Med UNAB 2004; 7(21):172-180 [Internet]. Disponible en: http://caribdis.unab.edu.co/pls/ portal/docs/PAGE/REVISTAMEDUNAB/NUMEROSANTERIORES/REVISTA721/RT_C2.PDF Consultado agosto del 2007. 